\title{
Effect of Different Concentrations of Fluoride in Dentifrices on Dentin Erosion Subjected or Not to Abrasion in situ/ex vivo
}

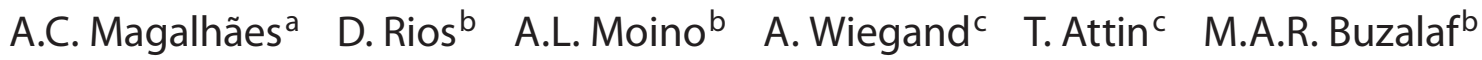 \\ ${ }^{a}$ Department of Pediatric and Social Dentistry, School of Dentistry of Araçatuba, São Paulo State University, \\ Araçatuba, ${ }^{b}$ Department of Oral Sciences, Bauru School of Dentistry, University of São Paulo, Bauru, Brazil; \\ 'Clinic for Preventive Dentistry, Periodontology and Cariology, University of Zurich, Zurich, Switzerland
}

\section{Key Words}

Erosion · Fluoride $\cdot$ Soft drinks $\cdot$ Toothbrushing abrasion

\section{Abstract}

This in situ/ex vivo study assessed the effect of different concentrations of fluoride in dentifrices on dentin subjected to erosion or to erosion plus abrasion. Ten volunteers took part in this crossover and double-blind study performed in 3 phases (7 days). They wore acrylic palatal appliances containing 4 bovine dentin blocks divided in two rows: erosion and erosion plus abrasion. The blocks were subjected to erosion by immersion ex vivo in a cola drink $(60 \mathrm{~s}, \mathrm{pH} 2.6) 4$ times daily. During this step, the volunteers brushed their teeth with one of three dentifrices $\mathrm{D}(5,000 \mathrm{ppm} \mathrm{F}, \mathrm{NaF}$, silica); C (1,100 ppm F, NaF, silica) and placebo (22 ppm F, silica). Then, the respective dentifrice slurry (1:3) was dripped on dentin surfaces. While no further treatment was performed in one row, the other row was brushed using an electric toothbrush for $30 \mathrm{~s}$ ex vivo. The appliances were replaced in the mouth and the volunteers rinsed with water. Dentin loss was determined by profilometry and analyzed by 2-way ANOVA/Bonferroni test $(\alpha=0.05)$. Dentin loss after erosive-abrasive wear was significantly greater than after erosion alone. Wear was significantly higher for the placebo than for the $D$ and $C$ dentifrices, which were not significantly different from each other. It can be concluded that the presence of fluoride concen-

\section{KARGER}

Fax +41613061234

E-Mail karger@karger.ch

www.karger.com
(C) 2008 S. Karger AG, Basel

$0008-6568 / 08 / 0422-0112 \$ 24.50 / 0$

Accessible online at:

www.karger.com/cre trations around 1,100 ppm in dentifrices is important to reduce dentin wear by erosion and erosion + abrasion, but the protective effect does not increase with fluoride concentration.

Copyright $\odot 2008$ S. Karger AG, Basel

Toothbrushing with fluoridated toothpaste is the most common method of oral hygiene and is responsible for the reduction of the prevalence of dental caries [Twetman et al., 2003]. However, clinical and laboratory studies suggest that this procedure can cause gingival trauma and consequently, recession and exposure of root dentin [Addy et al., 2002]. The exposure of dentin might be accompanied by an increased risk for dentin wear by different physical and chemical processes.

Erosion is becoming a common problem in modern societies, through the increased consumption of acid drinks, such as soft drinks, sport drinks, fruit juices and fruit teas [Lussi et al., 2004]. The acidic attack leads to irreversible loss of dental hard tissue, which is accompanied by a progressive softening of the surface [Amaechi and Higham, 2001]. This softened zone is more susceptible to mechanical forces, such as abrasion [Jaeggi and Lussi, 1999; Attin et al., 1998, 2000, 2001; Eisenburger et al., 2003; Rios et al., 2006; Magalhães et al., 2007], which in turn have little or no effect on sound dental hard tis- 
sues [Addy and Hunter, 2003]. In the clinical situation, toothbrushing seems to have major effects on acid-softened hard substances such as eroded enamel [Attin et al., 2001] or dentin [Wiegand et al., 2006] as well as carieslike enamel lesions [Kielbassa et al., 2005].

Several factors are associated with toothbrushing abrasion. Toothpaste characteristics, such as abrasivity, $\mathrm{pH}$, buffer capacity and fluoride content seem to be mainly relevant for the abrasion of dental hard tissues [Wiegand et al., 2004]. While some in vitro and in situ studies showed that the application of highly concentrated fluoride gels or solutions might be effective in decreasing erosion of dentin [Ganss et al., 2004a, b], only limited information about the impact of fluoridated dentifrices is available as yet. Some studies showed a limited beneficial effect of commercial fluoride dentifrice $(1,000-1,500$ ppm F) compared to nonfluoridated dentifrices on erosion and abrasion [Ponduri et al., 2005; Magalhães et al., 2007].

The beneficial effect of fluoride is associated with formation of a $\mathrm{CaF}_{2}$-like precipitate. This $\mathrm{CaF}_{2}$-like layer might be increased by application of more highly concentrated fluoride agents [ten Cate, 1999]. Thus, it would be interesting to determine whether a high-fluoride dentifrice $(5,000 \mathrm{ppm} \mathrm{F})$ might decrease dentin wear, and the purpose of this in situ study was to assess the effect of commercial dentifrices with different fluoride concentrations on dentin subjected to erosion or to erosion plus abrasion using an in situ protocol. The null hypotheses tested were: (1) there is no difference between erosion and erosion plus abrasion regarding dentin wear; (2) 5,000 ppm fluoride dentifrice is not able to reduce dentin wear by erosion and erosion plus abrasion in comparison to 1,100 ppm fluoride dentifrice and (3) 1,100 ppm fluoride dentifrice is not able to reduce dentin wear by erosion and erosion plus abrasion compared to placebo (no fluoride) dentifrice.

\section{Material and Methods}

\section{Experimental Design}

This study was approved by the Research and Ethics Committee of the Bauru School of Dentistry, University of São Paulo (Proc. No. 006/2007). It was a crossover and double-blind study performed in three phases of 7 days each, with a washout period of 7 days between the phases. Ten healthy adult volunteers ( $8 \mathrm{fe}-$ males and 2 males, aged 19-30 years) residing in the same fluoridated area $(0.70 \mathrm{mg} \mathrm{F} / \mathrm{l})$, who fulfilled the inclusion criteria described below, took part in this study. They presented normal salivary parameters, such as adequate stimulated and unstimulated salivary flows $(1.88 \pm 1.00$ and $0.60 \pm 0.33 \mathrm{ml} / \mathrm{min}$, respec- tively) and salivary $\mathrm{pH}(7.32 \pm 0.30)$. The subjects were free from erosive lesions or untreated carious cavities. The number of volunteers in the present study was defined according to previous studies [Rios et al., 2006; Magalhães et al., 2007]. They wore acrylic palatal appliances, which contained 4 bovine dentin blocks divided in two rows: erosion and erosion plus abrasion. The use of two conditions in the same intraoral palatal appliance was supported by the absence of a cross-effect in previous studies [Rios et al., 2006; Magalhães et al., 2007]. The tested dentifrices were: D $(5,397 \pm 136$ ppm F, NaF, silica, RDA $77 \pm 11, \mathrm{pH} 8.5$, Duraphat; Colgate, USA); C (1,042 \pm 58 ppm F, NaF, silica, RDA 100, pH 7.3, Crest; Procter and Gamble, USA) and placebo (22 \pm 1 ppm F, silica, RDA $77 \pm 11, \mathrm{pH} 8.5$, Duraphat; Colgate, USA). The mean total fluoride concentrations of the agents were checked prior to the in situ phase using a specific electrode (Orion 9609, Orion Research., Beverly, Mass., USA) according to Bardal et al. [2003]. The $\mathrm{pH}$ was measured in the dentifrice slurries. RDA values for D and C were reported by Barbakow et al. [1989] and Rice et al. [2001], respectively.

Erosion was performed with cola drink ( $\mathrm{pH} 2.6,60 \mathrm{~s}$ ) and the abrasion by toothbrushing with respective dentifrice slurry (30 s), 4 times a day. After each phase, dentin loss was determined by profilometry.

\section{Dentin Samples and Palatal Appliance Preparation}

One hundred and twelve root dentin blocks $(4 \times 4 \times 3 \mathrm{~mm})$ were prepared from extracted bovine incisors, which had been disinfected by storage in $2 \%$ formaldehyde solution $(\mathrm{pH} 7.0)$ for 30 days at room temperature [White, 1987]. Two blocks were obtained from the cervical surface of each root, by a cutting machine (Isomet Low Speed Saw, Buehler Ltda., Lake Bluff, USA) and two diamond disks (Extec Corp., Enfield, USA), which were separated by a $4-\mathrm{mm}$ diameter spacer, perpendicularly to the root dentin surface. The labial dentin surface of the blocks was ground flat with water-cooled carborundum discs $(320,600$ and 1,200 grades of $\mathrm{Al}_{2} \mathrm{O}_{3}$ papers; Buehler, Lake Bluff, USA), and polished with felt paper wet with $1 \mu \mathrm{m}$ diamond spray (Buehler). This procedure resulted in a removal of about $200 \mu \mathrm{m}$ of the outermost layer as checked with a micrometer (Digimatic, Mitutoyo, Tokyo, Japan). The complete removal of cementum was checked microscopically $(40 \times$ magnification). For allocation of the samples to the groups, the surface microhardness was determined by performing five indentations in different regions of the samples (Knoop diamond, 10 g, 5 s, HMV-2000; Shimadzu Corporation, Tokyo, Japan). The overall range of microhardness was 55-75 KHN. Specimens were allocated to treatments by stratified randomization according to the mean surface microhardness. All groups presented the same mean microhardness $(65 \pm 10 \mathrm{KHN})$. In order to maintain reference surfaces for lesion depth determination by profilometry, two layers of nail varnish were applied on half of the surface of each sample. Each two specimens were arranged in one row on the left and right sides of the appliance. For 5 volunteers, erosion was performed on the right and erosion + abrasion on the left side; in the other 5 volunteers, erosion was performed on the left row and the erosion + abrasion on the right row.

\section{Treatments}

Seven days prior to the beginning and throughout the experimental phase (7 days), the volunteers brushed their teeth with one of the respective dentifrices. In this crossover protocol, the volun- 
Table 1. Wear $(\mu \mathrm{m})$ of dentin blocks subjected to erosion or erosion + abrasion in the presence of different dentifrices

\begin{tabular}{llll}
\hline Dentifrice & Condition & $\begin{array}{l}\text { Mean difference } \\
\text { (erosion + abrasion) - erosion }\end{array}$ \\
\cline { 2 - 3 } & erosion $^{\mathrm{A}}$ & ${\text { erosion }+ \text { abrasion }^{\mathrm{B}}}$ & \\
\hline Placebo & $3.58 \pm 0.71^{\mathrm{a}}(3.13-4.22)$ & $3.89 \pm 1.09^{\mathrm{a}}(3.17-4.74)$ & $0.30 \pm 0.81^{\mathrm{a}}(-0.28-0.88)$ \\
1,100 ppm F & $2.45 \pm 0.50^{\mathrm{b}}(2.09-2.81)$ & $2.92 \pm 0.62^{\mathrm{b}}(2.48-3.37)$ & $0.48 \pm 0.70^{\mathrm{a}}(-0.02-0.97)$ \\
5,000 ppm F & $2.58 \pm 0.80^{\mathrm{b}}(2.04-3.14)$ & $2.88 \pm 0.89^{\mathrm{b}}(2.24-3.51)$ & $0.29 \pm 0.46^{\mathrm{a}}(0.61-0.04)$ \\
\hline
\end{tabular}

Figures are expressed as means \pm SD $(95 \% \mathrm{CI})$. Between columns, different upper-case superscript letters indicate significant difference between conditions. Within columns, different lower-case superscript letters indicate significant differences among the dentifrices $(\mathrm{p}<0.01)$.

teers were randomly allocated to the treatments and participated in 3 phases. In the first $12 \mathrm{~h}$ of each intraoral phase, specimens were not subjected to erosive and abrasive treatment to allow the formation of a salivary pellicle [Hara et al., 2006]. On the following 7 days, erosive and abrasive challenges were made extraorally 4 times a day at predetermined times $(8: 00,12: 00,16: 00$ and 20:00 h) after the mean meals.

For erosion of the dentin samples, the volunteers were instructed to remove the appliance and immerse it in a cup containing $150 \mathrm{ml}$ of a freshly opened bottle of regular Coke ( $\mathrm{pH} 2.6$; Coca-cola Company Spal, Porto Real, Brazil) at room temperature for $60 \mathrm{~s}$. During this ex vivo erosion, the volunteers brushed their teeth with one of the dentifrices using a soft end-rounded toothbrush (Sorriso Infantil, Brazil) with a small portion of the dentifrice (approximately $0.3 \mathrm{~g}$ ). After erosion of the blocks, 1 drop (around $35 \mu \mathrm{l}$ ) of the dentifrice slurry (dentifrice:water ratio, 1:3) was dripped on the dentin surface of each sample. While no treatment was performed in one row, the other row was brushed using a soft end-rounded electric toothbrush (Colgate Montions Multi-action, Brazil) for $30 \mathrm{~s}$ (166 oscillations/s) ex vivo. Volunteers were trained and instructed to carefully perform this procedure and to avoid a carry-across effect of the treatments. The appliances were replaced into the mouth and the volunteers rinsed with water $(10 \mathrm{ml}, 5 \mathrm{~s})$.

All solutions and dentifrices used by the volunteers were placed in separate vials which did not allow their identification in order to conform to the blind protocol of the study. The volunteers received instructions to wear the appliances continuously for $24 \mathrm{~h}$ but to remove them during meals ( 4 times a day, $1 \mathrm{~h}$ each). In this period, the appliance was stored in wet gauze. They were also instructed to not touch the tongue on the appliance, in order to avoid abrasion of the samples [Gregg et al., 2004]. The volunteers received oral and written information to refrain from using any fluoridated product.

\section{Dentin Loss Measurement}

After 7 days, the dentin blocks were removed from the appliances and the nail varnish on the reference surfaces was carefully removed with acetone-soaked cotton wool [Attin et al., 2000]. The dentin samples were maintained wet until the analysis to avoid shrinkage. Surface profiles of the dentin samples were obtained with a stylus profilometer (Mahr Perthometer, Göttingen, Ger- many). For determination of dentin loss, four profiles were recorded across the protected and eroded surfaces. One line was recorded on the protected surface only and served as baseline control for determination of dentin loss. The profile scans were performed in the center of each specimen at intervals of about 250 $\mu \mathrm{m}$. Control and eroded areas scans were superimposed and the average depth of the area under the curve in the eroded area was calculated with specially designed software. The results of the four scans were averaged for each specimen.

\section{Statistical Analysis}

GraphPad Prism software 4 version 4.0 for Windows (Graph Pad Software; San Diego, Calif., USA) was used. The assumptions of equality of variances and normal distribution of errors were checked for all the variables tested. Since the assumptions were satisfied, two-way repeated-measures ANOVA and Bonferroni post-hoc test were used. The mean difference between erosiveabrasive wear and erosive wear was tested using one-way ANOVA. The significance level was set at $5 \%$.

\section{Results}

Two-way repeated-measures ANOVA revealed a significant difference between the conditions ( $p=0.007)$, as well as among the dentifrices $(p=0.004)$. The interaction between the factors was not significant $(\mathrm{p}=0.794)$. Table 1 shows that for both erosion and erosion-abrasion, wear was significantly higher for the placebo dentifrice than for the D and C dentifrices $(\mathrm{p}<0.01)$, but D and C did not differ from each other $(p>0.05)$. Erosive-abrasive wear was significantly higher than wear through erosion alone $(p<0.01)$. There was no significant difference among the dentifrices in the difference between erosiveabrasive wear and erosive wear alone (one-way ANOVA; $\mathrm{p}=0.78$ ).

Based on the results, the first and third null hypotheses were rejected, while the second was accepted. 


\section{Discussion}

Although fluoridated dentifrices are the most frequently used topical fluoride agents, to date only three studies have focused on their effect on erosion and abrasion. Bartlett et al. [1994] found that fluoridated toothpaste had no effect on erosion plus abrasion compared to placebo dentifrice. Ponduri et al. [2005] concluded that brushing softened dentin with fluoridated toothpaste led to less tissue loss than brushing with nonfluoridated toothpaste. Magalhães et al. [2007] reported similar findings for enamel. However, in these studies, the preventive effect of fluoridated dentifrice on enamel and dentin wear by erosion plus abrasion was limited.

We tested the effect of a high-fluoride dentifrice on dentin wear. The in situ/ex vivo protocol was chosen to simulate the in vivo situation as closely as possible. This model allowed the formation of an acquired salivary pellicle which might play an important role during the erosive challenge and influence the interaction between fluoride and mineral [Hara et al., 2006]. Erosion was produced by a cola drink, as it is one of the most widely consumed soft drinks and exhibits erosive potential [Rios et al., 2006; Magalhães et al., 2007].

Softening was performed by extraoral immersion in cola for $60 \mathrm{~s}$, which is assumed to be representative of the effects after rapid consumption of an acidic beverage. Brushing abrasion of each two specimens (one row) was performed for $30 \mathrm{~s}$ each cycle (15 s per specimen). Thus, the toothbrushing time of one specimen slightly exceeded the contact time reported by Dyer et al. [2000] and Hooper et al. [2003]. The last authors assumed a contact time for one tooth of $10 \mathrm{~s}$ during twice daily toothbrushing.

The erosive and abrasive treatments were performed ex vivo to avoid damage to the volunteers' teeth and to avoid cross-effects between the treatments. Therefore, the applied challenges were not immediately influenced by saliva. The volunteers avoided contact between the tongue and the appliance, in order to minimize abrasion of the samples, although Gregg et al. [2004] showed that wear of eroded dentin could not be increased significantly by licking. These aspects have to be carefully considered when the results are extrapolated to the in vivo situation. It is probable that in vivo the dentin wear is reduced when compared to the present study.

The results confirm previous studies showing that abrasion can increase wear of acid-softened tooth surfaces. Studies have shown that erosive demineralization results in the formation of an outer layer of a fully demineralized organic matrix followed by a partly demineral-

Effect of Fluoridated Dentifrice on

Dentin Wear ized zone until the sound inner dentin is reached [Kinney et al., 1995; Ganss et al., 2001]. The dentin demineralization rate decreases when the amount of degradable collagen increases, and the demineralized matrix is believed to hamper ionic diffusion into and out of the demineralizing area [Ganss et al., 2004b]. The demineralized layer of matrix could have buffering effects sufficient to retard demineralization and to influence the effectiveness of fluoride. As erosive lesions do not present a subsurface lesion available for remineralization, the action of fluoride is mainly attributed to a precipitation of $\mathrm{CaF}_{2}$-like material on eroded dental surfaces [Ganss et al., 2004a, b]. Ganss et al. [2004b] demonstrated in vitro that the presence of the dentin matrix is essential for the effectiveness of fluoride in decreasing dental erosion. In the in situ situation, salivary enzymes can degrade collagen layers exposed by complete erosive demineralization [Klont and ten Cate, 1991]. However, the clinical significance of salivary collagenase levels still has to be clarified. Besides, it is necessary to test the resistance of collagen-rich layers to mechanical forces such as toothbrushing.

Both fluoridated dentifrices reduced erosive and erosive-abrasive dentin wear by approximately $27.5 \%$ compared to the placebo toothpaste, but their efficacies were not significantly different. This might be explained by the use of diluted dentifrices (1:3 slurry) which might have diminished the formation of $\mathrm{CaF}_{2}$. This protocol, however, was chosen to closely mimic the clinical performance of the commercial dentifrices, since they are diluted by saliva during toothbrushing. The application of undiluted dentifrices to the surfaces, as was done by Magalhães et al. [2007], could have increased the abrasive effect of brushing, but would also be expected to enhance the formation of $\mathrm{CaF}_{2}$. It should be taken into consideration that the mean differences in erosive-abrasive and erosive wear between the dentifrices were not significant. One might infer from this result that the effect of $\mathrm{F}$ dentifrices on erosive-abrasive wear is due more to the prevention of surface softening by erosion, than to the reduction of the abrasion of eroded enamel.

It is also possible that the amount of collagen present on the eroded dentin surface was too small - because of the short erosive challenge or because of degradation by salivary enzymes and abrasive forces - to allow the detection of significant differences between the $\mathrm{F}$ dentifrices. According to Ganss et al. [2004a], the buffering effect of the demineralized matrix layer reduces the $\mathrm{pH}$ fall within the layer; combined with the presence of high concentrations of fluoride, this leads to reduced demineralization. In the study by Ganss et al. [2004a], toothpaste fluo- 
ridation and intensive fluoridation (mouthrinse, $\mathrm{SnF}_{2}$, $0.025 \% \mathrm{~F}$ or gel, $\mathrm{NaF}, 1.25 \% \mathrm{~F}$ ) reduced the erosive mineral loss by 30 and $60 \%$, respectively. As these results were conducted in human coronal dentin, the efficacy of the $5,000 \mathrm{ppm} F$ dentifrice in human instead of bovine dentin should be evaluated in further studies.

From the results of the present study, it can be concluded that the presence of fluoride concentrations around 1,100 ppm in dentifrices can reduce but not inhibit dentin wear by erosion and erosion plus abrasion. In addition, the efficacy of $\mathrm{F}$ dentifrice does not increase with $\mathrm{F}$ concentration.

\section{Acknowledgments}

The authors would like to thank The State of São Paulo Research Foundation for the concession of a scholarship to the third author (Proc. 06/07260-4) and Diane Cummins, Colgate-Palmolive, Piscataway, N.J. for providing the placebo dentifrices for this study. We also thank the volunteers that took part in this study.

\section{References}

Addy M, Hughes J, Pickles MJ, Joiner A, Huntington E: Development of a method in situ to study toothpaste abrasion of dentine. Comparison of 2 products. J Clin Periodontol 2002;29:896-900

Addy M, Hunter ML: Can tooth brushing damage your health? Effects on oral and dental tissues. Int Dent J 2003;53:177-186.

-Amaechi BT, Higham SM: Eroded enamel lesion remineralization by saliva as a possible factor in the site-specificity of human denta erosion. Arch Oral Biol 2001;46:697-703.

-Attin T, Buchalla W, Gollner M, Hellwig E: Use of variable remineralization periods to improve the abrasion resistance of previously eroded enamel. Caries Res 2000;34:48-52.

-Attin T, Knöfel S, Buchalla W, Tütüncü R: In situ evaluation of different remineralization periods to decrease brushing abrasion of demineralized enamel. Caries Res 2001;35: 216-222.

-Attin T, Zirkel C, Hellwig E: Brushing abrasion of eroded dentin after application of sodium fluoride solutions. Caries Res 1998;32:344350.

Barbakow F, Imfeld T, Lutz F, Stookey G, Schemehorn B: Dentin abrasion (RDA), enamel abrasion (REA) and polishing scores of dentifrices sold in Switzerland. Schweiz Monatsschr Zahnmed 1989;99:408-413.

Bardal PAP, Olympio KPK, Cardoso VES, Bastos JRM, Buzalaf MAR: Evaluation of $\mathrm{pH}$ and total, soluble and ionic fluoride concentrations of dentifrices commercially available in Brazil. Oral Health Prev Dent 2003;1:283289.

Bartlett DW, Smith BGN, Wilson RF: Comparison of the effect of fluoride and non-fluoride toothpaste on tooth wear in vitro and the influence of enamel fluoride concentration and hardness of enamel. Brit Dent J 1994;76: 346-348.

Dyer D, Addy M, Newcombe RG: Studies in vitro of abrasion by different manual toothbrush heads and a standard toothpaste. J Clin Periodontol 2000;27:99-103.
Eisenburger M, Shellis RP, Addy M: Comparative study of wear of enamel induced by alternating and simultaneous combinations of abrasion and erosion in vitro. Caries Res 2003;37:450-455.

-Ganss C, Klimek J, Brune V, Schumann A: Effects of two fluoridation measures in erosion progression on enamel and dentine in situ. Caries Res 2004a;38:561-566.

Ganss C, Klimek J, Schäffer U, Spall T: Effectiveness of two fluoridation measures on erosion progression in human enamel and dentine in vitro. Caries Res 2001;35:325-330.

Ganss C, Klimek J, Starck C: Quantitative analysis of the impact of the organic matrix on the fluoride effect on erosion progression in human dentine using longitudinal microradiography. Arch Oral Biol 2004b;49:931-935.

Gregg T, Mace S, West NX, Addy M: A study in vitro of the abrasive effect of the tongue on enamel and dentine softened by acid erosion. Caries Res 2004;38:557-560.

Hara AT, Ando M, Gonzalez-Cabezas C, Cury JA, Serra MC, Zero DT: Protective effect of the dental pellicle against erosive challenges in situ. J Dent Res 2006;85:612-616.

Hooper S, West NX, Pickles MJ, Joiner A, Newcombe RG, Addy M: Investigation of erosion and abrasion on enamel and dentine: a model in situ using toothpastes of different abrasivity. J Clin Periodontol 2003;30:802-808.

Jaeggi T, Lussi A: Toothbrush abrasion of erosively altered enamel after intraoral exposure to saliva: an in situ study. Caries Res 1999;33:455-461.

Kielbassa AM, Gillmann L, Zantner C, MeyerLueckel H, Hellwig E, Schulte-Monting J: Profilometric and microradiographic studies on the effects of toothpaste and acidic gel abrasivity on sound and demineralized bovine dental enamel. Caries Res 2005;39:380386.

Kinney SH, Balooch M, Haript DL Jr, Marshall SJ, Marshall GW Jr: Mineral distribution and dimensional changes in human dentin during demineralization. J Dent Res 1995; 74:1179-1184.
Klont B, ten Cate JM: Remineralisation of bovine incisor root lesions in vitro: the role of the collagenous matrix. Caries Res 1991;25:39-45.

Lussi A, Jaeggi T, Zero D: The role of diet in the aetiology of dental erosion. Caries Res 2004:38:34-44.

Magalhães AC, Rios D, Delbem ACB, Buzalaf MAR, Machado MAAM: Influence of fluoride dentifrice on brushing abrasion of eroded human enamel: an in situ/ex vivo study. Caries Res 2007;41:77-79.

Ponduri S, Macdonald E, Addy M: A study in vitro of the combined effects of soft drinks and tooth brushing with fluoride toothpaste on the wear of dentine. Int J Dent Hyg 2005; 3:7-12.

Rice DE, Dhabhan DJ, White DJ: Laboratory stain removal and abrasion characteristics of a dentifrice based upon a novel silica technology. J Clin Dent 2001;12:34-37.

Rios D, Honório HM, Magalhães AC, Delbem AC, Machado MAAM, Silva SMB et al: Effect of salivary stimulation on erosion subjected or not to abrasion in human and bovine enamel: an in situ/ex vivo study. Caries Res 2006;40:218-223.

ten Cate JM: Current concepts on the theories of the mechanism of action of fluoride. Acta Odontol Scand 1999;57:325-329.

- Twetman S, Axelsson S, Dahlgren H, Holm AK, Källestal C, Lagerlöf F et al: Caries-preventive effect of fluoride toothpaste: a systematic review. Acta Odontol Scand 2003;61: 347-355.

White DJ: Reactivity of fluoride dentifrices with artificial caries 1. Effects on early lesions: $F$ uptake, surface hardening and remineralisation. Caries Res 1987;21:126-140.

Wiegand A, Lemmrich F, Attin T: Influence of rotating-oscillating, sonic and ultrasonic action of power toothbrushes on abrasion of sound and eroded dentine. J Periodontol Res 2006;41:221-227.

Wiegand A, Wolmershäuser S, Hellwig E, Attin T: Influence of buffering effects of dentifrices and fluoride gels on abrasion on eroded dentine. Arch Oral Biol 2004;49:259-265. 